RIMS-787-SPhT-91/140 (corrected)

\title{
Root Systems and Purely Elastic S-Matrices II
}

\author{
Patrick Dorey ${ }^{\dagger}$ \\ Service de Physique Théorique de Saclay, \\ 91191 Gif-sur-Yvette cedex, France
}

\begin{abstract}
Starting from a recently-proposed general formula, various properties of the ADE series of purely elastic S-matrices are rederived in a universal way. In particular, the relationship between the pole structure and the bootstrap equations is shown to follow from properties of root systems. The discussion leads to a formula for the signs of the three-point couplings in the simply-laced affine Toda theories, and a simple proof of a result due to Klassen and Melzer of relevance to Thermodynamic Bethe Ansatz calculations.
\end{abstract}

August 1991

$\dagger$ dorey@poseidon.saclay.cea.fr

1 Laboratoire de la Direction des Sciences de la Matière du Commissariat à l'Energie Atomique 


\section{Introduction}

Occasionally, an integrable perturbation of a conformal field theory results in a massive scattering theory which is purely elastic, in that the S-matrix is diagonal in a suitable basis [1]. This observation has lead to some work on such S-matrices as interesting objects in their own right. A series of examples connected with the ADE series of Lie algebras has been uncovered, both directly in the context of perturbed conformal field theory [2], and also via the study of affine Toda field theories [3 9] (in fact, there are slight differences between the S-matrices found in the two contexts; these will be mentioned where relevant). As is often the case in the study of integrable quantum field theories, the proposed Smatrices have not (at least so far) been derived from first principles, but rather deduced on the basis of certain assumptions and consistency requirements. However, for a theory with a diagonal S-matrix, the Yang-Baxter equation - often a very powerful tool in the study of the S-matrices of integrable theories [10] - is trivially satisfied, and so gives no information. There does remain the possibility that two particles in the theory may fuse to form a third as a bound state [11]. As emphasised by Zamolodchikov [1], for purely elastic scattering the resulting bootstrap equations are sufficiently simple to provide a useful set of consistency conditions, constraining both the conserved charges and the S-matrix.

The nature of the bootstrap solutions for the ADE theories is in fact closely linked to properties of the corresponding root systems, and in particular the action on these root systems of the Coxeter element of the Weyl group [12]. The aim of this paper is to explore this connection a little further, with particular emphasis on the implications for the structure of the S-matrix elements. By refining the notations used in [12], it turns out to be possible to streamline the discussion considerably. After a description of some necessary formulae, section two outlines how this goes. While this section contains no new results, it does give a proof of the S-matrix bootstrap equations which simplifies and clarifies that given previously. Section three is devoted to a discussion of the pole structure of S-matrix, and shows how this is exactly in accordance with the bound-state structure predicted by the fusings. Various empirically observed features of the purely elastic S-matrices turn out to be simple consequences of the properties of root systems. An application for some of the calculations in section three is given in section four, giving a description of one set of signs for the three-point couplings of the simply-laced affine Toda theories, in terms of roots and weights. Finally, section five gives a universal proof of an elegant formula due to Klassen and Melzer [9], and section six contains some concluding remarks. 


\section{Preliminaries}

Since this paper is a direct sequel to [12], the reader is referred back to that paper for details of the motivations and many of the original formulae. As mentioned in the introduction, elements of the discussion given there become rather clearer if the notation (in particular, the labelling of the orbits of the Coxeter element), is changed slightly. For this, some results to be found in a paper by Kostant [13] will be needed, and this section starts with a brief review of the relevant material.

First though, note that various of the definitions and results to be given in this section work equally well for simply-laced and non simply-laced root systems. For example, the discussion in [13] makes no distinction between the two cases. It is also worth noting that the discussion in [12] of the conserved charge bootstrap goes through essentially unchanged for the non simply-laced root systems. However, the S-matrix formulae given in that paper seem to be hard to generalise beyond the ADE series, possibly reflecting the difficulties that were found in the (quantum) problem of finding S-matrices for the non simply-laced affine Toda theories [4,7]. For this paper, then, attention will be restricted to the alreadyknown purely elastic scattering theories, that is to those associated with the simply-laced Lie algebras. The discussion will be based on a simply-laced root system $\Phi$, of rank $r$, with $\left\{\alpha_{i}\right\}$ a set of simple roots. Letting $w_{i}$ denote the Weyl reflection corresponding to the simple root $\alpha_{i}\left(\right.$ so $\left.w_{i}(x)=x-\frac{2}{\alpha_{i}^{2}}\left(\alpha_{i}, x\right) \alpha_{i}\right)$, set

$$
w=w_{1} w_{2} \ldots w_{r}
$$

so that $w$ is a Coxeter element. Also, let $\langle w\rangle$ be the subgroup of $W$, the Weyl group, generated by $w$. Finally, for $i=1 \ldots r$ define a root $\phi_{i}$ by

$$
\phi_{i}=w_{r} w_{r-1} \ldots w_{i+1}\left(\gamma_{i}\right) .
$$

Then the following results are given in [13]:

(i) With the definition of positive and negative roots implied by the given choice of simple roots, $\phi_{i}>0$ and $w\left(\phi_{i}\right)<0$.

(ii) If $\alpha$ is a root such that $\alpha>0$ and $w(\alpha)<0$, then $\alpha$ is one of the $\phi_{i}$.

(iii) Let $\Gamma_{i}$ be the orbit of $\phi_{i}$ under $\langle w\rangle$. Then the $\Gamma_{i}$ 's are disjoint, each has $h$ elements, and thus their union is all of $\Phi$. 
The set $\left\{\phi_{i}\right\}$ possesses one further useful property, which can be found for example in [14]. If $\lambda_{i}$ is the fundamental weight corresponding to the simple root $\alpha_{i}$, then

$$
\phi_{i}=\left(1-w^{-1}\right) \lambda_{i}
$$

(To prove this result, note that $w_{i}\left(\lambda_{j}\right)=\lambda_{j}-\delta_{i j} \alpha_{j}$, which follows since the fundamental weights are dual to the simple co-roots $\alpha_{i}^{\vee} \equiv \frac{2}{\alpha_{i}^{2}} \alpha_{i}$. For the simply-laced cases of interest here, $\alpha_{i}^{2}=2$ and the $\lambda_{i}$ are dual to the simple roots themselves.) This relation can be inverted, $w$ having no eigenvalue equal to one. Writing

$$
\left(1-w^{-1}\right)^{-1}=R
$$

it is easily checked that

$$
R=\frac{1}{h} \sum_{1}^{h} p w^{p}=-\frac{1}{h} \sum_{p=0}^{h-1} p w^{-p} .
$$

This mapping is not orthogonal, but rather satisfies

$$
(R \alpha, \beta)+(\alpha, R \beta)=(\alpha, \beta)
$$

Other identities, such as $(R \alpha, \beta)=-(w \alpha, R \beta)$, can also be found but only (2.5) will be used below. Projectors onto the various eigenspaces of $w$ are given by

$$
P_{s}=\frac{1}{h} \sum_{p=0}^{h-1} \omega^{-s p} w^{p}=\frac{1}{h} \sum_{p=0}^{h-1} \omega^{s p} w^{-p}
$$

with corresponding eigenvalues $\omega^{s}=e^{2 \pi i s / h}$. If $s$ is not an exponent of the algebra, then the spin $s$ eigenspace is null, and $P_{s}=0$.

It is often useful to focus on a particular ordering of the simple roots, linked to a twocolouring of the Dynkin diagram. This ordering is such that $\left\{\alpha_{i}\right\}$ splits into two subsets, each of which contains only mutually orthogonal roots:

$$
\left\{\alpha_{1}, \alpha_{2}, \ldots \alpha_{r}\right\}=\left\{\alpha_{1}, \ldots \alpha_{k}\right\} \cup\left\{\alpha_{k+1}, \ldots \alpha_{r}\right\}
$$

Defining subsets of the indices $1, \ldots r$ as

$$
\bullet=\{1,2, \ldots k\}, \quad \circ=\{k+1, k+2, \ldots r\}
$$


('black' and 'white'), the internal orthogonality amounts to the requirement that for $i \neq j$, $\left(\alpha_{i}, \alpha_{j}\right)=0$ if $i$ and $j$ have the same colour. By a small abuse of notation, the symbols $\bullet, \bullet^{\prime}, \circ, o^{\prime}$ and so on will occasionally be used to denote arbitrary indices taken from the corresponding (black or white) subset. A slightly different notation was used in [12]: black simple roots were called 'type alpha', white ones 'type beta'.

In this ordering,

$$
w=w_{\{\bullet\}} w_{\{\circ\}}
$$

with

$$
w_{\{\bullet\}}=\prod_{i \in \bullet} w_{i}, \quad w_{\{0\}}=\prod_{j \in \circ} w_{j} .
$$

The internal orthogonality of the two subsets of the simple roots implies that the reflections for simple roots of the same colour commute, and that a reflection for a given simple root will leave invariant all other simple roots of the same colour. It follows that

$$
\begin{aligned}
& \phi_{\bullet}=w_{\{\circ\}}\left(\alpha_{\bullet}\right), \\
& \phi_{\circ}=\alpha_{\circ} .
\end{aligned}
$$

In [12], the coset representatives were $\alpha_{\bullet}$ and $-\alpha_{\circ}$; since both $w_{\{\circ\}}$ and -1 induce charge conjugation on the cosets, this means that, strictly speaking, the assignment of cosets to simple roots induced by the $\phi_{i}$ differs by an overall charge conjugation from that used in the earlier paper. However this is merely a matter of labelling convention - for example, one can interchange the values of the conserved charges on particle and antiparticle simply by negating the normalisations of the even spin charges - and so can be ignored.

The ADE S-matrices will be built as products of functional 'building blocks'. In [12], the blocks used were

$$
\{x\}_{+}= \begin{cases}(x-1)_{+}(x+1)_{+} & \text {(perturbed conformal) } \\ \frac{(x-1)_{+}(x+1)_{+}}{(x-1+B)_{+}(x+1-B)_{+}} & (\text {affine Toda })\end{cases}
$$

where

$$
(x)_{+}=\sinh \left(\frac{\theta}{2}+\frac{i \pi x}{2 h}\right)
$$

and

$$
B(\beta)=\frac{1}{2 \pi} \frac{\beta^{2}}{1+\beta^{2} / 4 \pi} .
$$


The block for an affine Toda theory contains an extra coupling-constant dependent part over the 'minimal' version suitable for perturbed conformal theories. This turns out to have no effect on the physical pole structure $(\beta$ being real, and $B(\beta)$ therefore between 0 and 2), and so it is reasonable to use the same notation for both cases. In fact, the discussion of pole structure will be a little more transparent if this block is swapped for another, namely

$$
\{x\}_{-}= \begin{cases}-\{-x\}_{+}^{-1} & (\text { perturbed conformal }) \\ \{-x\}_{+}^{-1} & (\text { affine Toda })\end{cases}
$$

Of course, any formula involving $\{x\}_{-}$can be immediately rewritten in terms of $\{x\}_{+}$.

One further piece of notation will be needed. For any pair of roots $\alpha, \beta \in \Phi$, an integer $u(\alpha, \beta)$ can be defined modulo $2 h$ via the following relations:

$$
\begin{array}{rl}
u(\alpha, \beta)=-u(\beta, \alpha) & u(w \alpha, \beta)=u(\alpha, \beta)+2 \\
u\left(\phi_{\bullet}, \phi_{\bullet^{\prime}}\right)=u\left(\phi_{\circ}, \phi_{\circ^{\prime}}\right)=0 & u\left(\phi_{\circ}, \phi_{\bullet}\right)=1 .
\end{array}
$$

For the coset representatives $\phi_{i}$, the abbreviated notation $u_{i j} \equiv u\left(\phi_{i}, \phi_{j}\right)$ will often be used. The definition is natural in that $\frac{\pi s}{h} u(\alpha, \beta)$ is the (signed) angle between the projections of the roots $\alpha$ and $\beta$ into the $\omega^{s}$ eigenspace of $w$. Now the three-point couplings are described by the following fusing rule [12] (also relevant in other contexts [15]):

$$
C^{i j k} \neq 0 \text { iff } \exists \operatorname{roots} \alpha_{(i)} \in \Gamma_{i}, \alpha_{(j)} \in \Gamma_{j}, \alpha_{(k)} \in \Gamma_{k} \text { with } \alpha_{(i)}+\alpha_{(j)}+\alpha_{(k)}=0 .
$$

(Note the use here of a convention that will be adhered to for the rest of this paper: $\alpha_{(i)}$, for example, is used for any root that lies in $G_{i}$, the orbit of the root $\phi_{i}$. It is important not to confuse this with the simple root $\alpha_{i}$-in particular, even though the labelling of the orbits ultimately derives from the choice of simple roots, via (2.1), there are cases where the simple root $\alpha_{i}$ does not lie in the orbit $\Gamma_{i}$.) Since the fusing angles $U_{i j}^{k}$ are the relative angles of projections into the $\omega^{1}$ eigenspace, they are related to the $u\left(\alpha_{(i)}, \alpha_{(j)}\right)$ by

$$
U_{i j}^{k}=\frac{\pi}{h}\left|u\left(\alpha_{(i)}, \alpha_{(j)}\right)\right|, \quad \text { where } \alpha_{(i)}+\alpha_{(j)} \in \Gamma_{\bar{k}}
$$

Being signed angles, the $u(\alpha, \beta)$ 's also satisfy

$$
u(\alpha, \beta)+u(\beta, \gamma)+u(\gamma, \alpha)=0 \bmod 2 h
$$

for (any) three roots $\alpha, \beta$ and $\gamma$. 
Armed with these conventions, the expression given in 12 for the two-particle Smatrix can be rewritten in a compact way:

$$
S_{i j}=\prod_{p=0}^{h-1}\left\{2 p+1+u_{i j}\right\}_{ \pm}^{\left(\lambda_{i}, w^{-p} \phi_{j}\right)} .
$$

Note, the expression is identical in form whether it is written in terms of $\{x\}_{+}$or $\{x\}_{-}$. This is equivalent to unitarity $\left(S_{i j}(\theta) S_{i j}(-\theta)=1\right)$ and follows from

$$
\left(\lambda_{i}, w^{-p} \phi_{j}\right)=-\left(\lambda_{i}, w^{p+1+u_{i j}} \phi_{j}\right) .
$$

Symmetry $\left(S_{i j}=S_{j i}\right)$ can also be checked, using

$$
\left(\lambda_{i}, w^{-p} \phi_{j}\right)=\left(\lambda_{j}, w^{-p-u_{i j}} \phi_{i}\right)
$$

(A 'mixed' equality, $\left(\lambda_{i}, w^{-p} \phi_{j}\right)=-\left(\lambda_{j}, w^{p+1} \phi_{i}\right)$, follows directly from (2.2), independently of the special root ordering (2.7).) Equivalent formulae were given in [12], but in a less compact way.

Equation (2.17) can be put into a perhaps more suggestive form by noting from (2.13) that $2 p+u_{i j}$ is just $u\left(\phi_{i}, w^{-p} \phi_{j}\right)$. Hence

$$
S_{i j}=\prod_{\alpha_{(j)} \in \Gamma_{j}}\left\{u\left(\phi_{i}, \alpha_{(j)}\right)+1\right\}_{ \pm}^{\left(\lambda_{i}, \alpha_{(j)}\right)} .
$$

The unitarity and symmetry of this formula can be checked directly by rewriting (2.18) as

$$
\begin{aligned}
& \left(\lambda_{i}, \alpha_{(j)}\right)=-\left(\lambda_{i}, w^{u\left(\phi_{i}, \alpha_{(j)}\right)+1} \alpha_{(j)}\right) \\
& \left(\lambda_{i}, \alpha_{(j)}\right)=\left(\lambda_{j}, w^{\left(u\left(\phi_{j}, \alpha_{(i)}\right)-u\left(\phi_{i}, \alpha_{(j)}\right)\right) / 2} \alpha_{(i)}\right) .
\end{aligned}
$$

Note, the exponent of $w$ in (2.20b) is an integer, since $u\left(\phi_{j}, \alpha_{(i)}\right)$ and $u\left(\phi_{i}, \alpha_{(j)}\right)$ are always either both even or both odd. The important case for the symmetry of $(2.19)$ is $\left(\lambda_{i}, \alpha_{(j)}\right)=$ $\left(\lambda_{j}, \alpha_{(i)}\right)$ if $u\left(\phi_{j}, \alpha_{(i)}\right)=u\left(\phi_{i}, \alpha_{(j)}\right)$.

The S-matrix bootstrap equations [1] can be checked very simply from (2.19). First these equations are rewritten, for each particle species $l$ and each nonvanishing three-point coupling $C^{i j k}$, as

$$
S_{l i}(\theta) S_{l j}\left(\theta+i U_{i j}^{k}\right) S_{l k}\left(\theta-i U_{i k}^{j}\right)=1 \text {. }
$$

(This alternative bootstrap equation, obtained from the more usual one via the equations of unitarity $\left(S_{i j}(\theta) S_{i j}(-\theta)=1\right)$ and crossing $\left(S_{i j}(\theta)=S_{i \bar{\jmath}}(i \pi-\theta)\right)$, is analogous to the 
symmetrical version of the conserved charge bootstrap equation used in [12].) The structure of this equation is clarified if a shift operator $\mathcal{T}_{y}$ is introduced, defined by

$$
\left(\mathcal{T}_{y} f\right)(\theta)=f\left(\theta+\frac{i \pi y}{h}\right)
$$

and acting on the blocks as

$$
\mathcal{T}_{y}\{x\}_{ \pm}=\{x \pm y\}_{ \pm}
$$

Recalling from (2.14) that $C^{i j k} \neq 0$ implies the existence of a root triangle $\left\{\alpha_{(i)}, \alpha_{(j)}, \alpha_{(k)}\right\}$, the relation (2.15) can be used to write equation (2.21) as

$$
\left(S_{l i}\right)\left(\mathcal{T}_{u\left(\alpha_{(i)}, \alpha_{(j)}\right)} S_{l j}\right)\left(\mathcal{T}_{u\left(\alpha_{(i)}, \alpha_{(k)}\right)} S_{l k}\right)=1
$$

Note how the fact that $u(\alpha, \beta)$ is a signed angle takes care of the relative minus sign between the two shifts in the earlier equation, (2.21). Of course, depending on the orientation of the projection of the root triangle into the $s=1$ subspace, there could be an overall negation of the shifts in (2.24) compared to (2.21). In fact, root triangles projecting to both orientations always exist - this will be discussed in more detail in section four - but this is not a problem since (2.21) also holds with the labels $j$ and $k$ exchanged, an operation which itself has the effect of negating the two shifts.

Finally, acting on both sides with $\mathcal{T}_{u\left(\phi_{l}, \alpha_{(i)}\right)}$ and using 2.16 gives

$$
\left(\mathcal{T}_{u\left(\phi_{l}, \alpha_{(i)}\right)} S_{l i}\right)\left(\mathcal{T}_{u\left(\phi_{l}, \alpha_{(j)}\right)} S_{l j}\right)\left(\mathcal{T}_{u\left(\phi_{l}, \alpha_{(k)}\right)} S_{l k}\right)=1 \quad\left(\alpha_{(i)}+\alpha_{(j)}+\alpha_{(k)}=0\right) .
$$

To verify that the bootstrap equations in this form are satisfied by (2.19) is almost immediate. The left hand side of (2.25) involves three roots running through the orbits $\Gamma_{i}, \Gamma_{j}$ and $\Gamma_{k}$ for the S-matrix elements $S_{l i}, S_{l j}$ and $S_{l k}$ respectively. If these orbits are labelled starting at $\alpha_{(i)}, \alpha_{(j)}$ and $\alpha_{(k)}$ (instead of the roots $\phi_{i}, \phi_{j}$ and $\phi_{k}$ that would have been used had (2.17) been the starting point), then via (2.23) the left hand side is

$$
\begin{aligned}
& \prod_{p=0}^{h-1}\left\{u\left(\phi_{l}, w^{-p} \alpha_{(i)}\right) \pm u\left(\phi_{l}, \alpha_{(i)}\right)+1\right\}_{ \pm}^{\left(\lambda_{l}, w^{-p} \alpha_{(i)}\right)} \\
& \quad \times\left\{u\left(\phi_{l}, w^{-p} \alpha_{(j)}\right) \pm u\left(\phi_{l}, \alpha_{(j)}\right)+1\right\}_{ \pm}^{\left(\lambda_{l}, w^{-p} \alpha_{(j)}\right)} \\
& \quad \times\left\{u\left(\phi_{l}, w^{-p} \alpha_{(k)}\right) \pm u\left(\phi_{l}, \alpha_{(k)}\right)+1\right\}_{ \pm}^{\left(\lambda_{l}, w^{-p} \alpha_{(k)}\right)}
\end{aligned}
$$

That this is equal to one is apparent if the $\{x\}_{-}$blocks have been used $\left(\{x\}_{+}\right.$being appropriate for the equivalent version of (2.25) with all shifts negated). From (2.13), for 
each value of $p$ the three blocks involved are then equal to the same function, namely $\{2 p+1\}_{-}$. The total power to which this is raised is $\left(\lambda_{i}, w^{-p}\left(\alpha_{(i)}+\alpha_{(j)}+\alpha_{(k)}\right)\right)$. But this is zero, since $\alpha_{(i)}, \alpha_{(j)}$ and $\alpha_{(k)}$ form a root triangle for the coupling $C^{i j k}$. Hence the whole expression is equal to one, as required.

Section five will give a general proof of a result for the minimal scattering theories due to Klassen and Melzer, for which it will be helpful to have an expression for the minimal S-matrix elements in terms of the unitary blocks

$$
(x)=(x)_{+} /(-x)_{+} \cdot
$$

(This block, unitary in the sense that it individually satisfies the unitarity constraint mentioned above, was used in [4], along with the larger unitary block $\{x\}=\{x\}_{+} /\left\{-_{-}\right\}_{+}$.) Via (2.9) and (2.26), the formulae (2.17) and (2.19) become, in the minimal cases,

$$
S_{i j}=\prod_{p=0}^{h-1}\left(2 p+u_{i j}\right)^{\left(\lambda_{i}, w^{-p} \phi_{j}\right)}=\prod_{\alpha_{(j)} \in \Gamma_{j}}\left(u\left(\phi_{i}, \alpha_{(j)}\right)\right)^{\left(\lambda_{i}, \alpha_{(j)}\right)} .
$$

\section{Pole structure}

This section will involve a detailed examination of the physical pole structure of Smatrices described by (2.17). The nature of the residues will be studied, for which a little more information on the building block $\{x\}$ - will be needed. This is a $2 \pi i$ periodic function, real for imaginary $\theta$. It has simple poles at $(x-1) \pi i / h$ and $(x+1) \pi i / h$, with residues positive multiples of $-i,+i$ respectively. Outside the interval between these two poles (or its repetition modulo $2 \pi i$ ), the function is positive on the imaginary axis. These facts hold equally well for the perturbed conformal or the affine Toda blocks.

It is helpful to introduce a pictorial notation in which the S-matrix element is represented by a 'wall' of rectangles, stacked along the imaginary axis. The building block $\{x\}_{-}$ is depicted by a single rectangle above the imaginary axis, stretching from $(x-1) \pi i / h$ to $(x+1) \pi i / h$ :

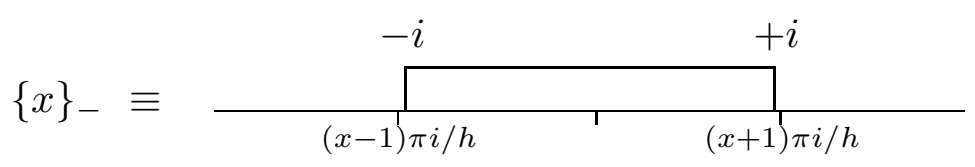


The residues of the poles at $(x \pm 1) \pi i / h$, up to some real, positive constant, are shown above the block. A product of blocks making up an S-matrix element is represented by stacking the rectangles to make a wall. For example,

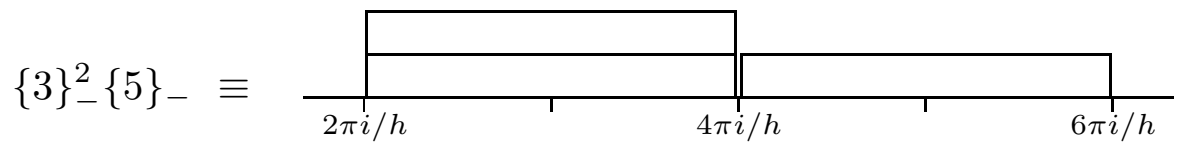

Poles occur at the ends of the blocks, of higher order where the ends of blocks coincide. In this example there is a pole of order three at $\theta=4 \pi i / h$, coming from the two blocks to the left and one to the right. To represent a full S-matrix element, a wall of length $2 \pi$ is sufficient, since $\{x+2 h\}_{-}=\{x\}_{-}$. In fact, the unitarity constraint imposes that the height for $\{-x\}_{-}$must be exactly the negative of that for $\{x\}_{-}$, so a stretch of length $\pi$ will do; it is convenient to let it straddle the physical strip, running from 0 to $i \pi$. To give a couple of examples, here are two of the S-matrix elements from the $E_{8}$-related scattering theories: $S_{35}:$

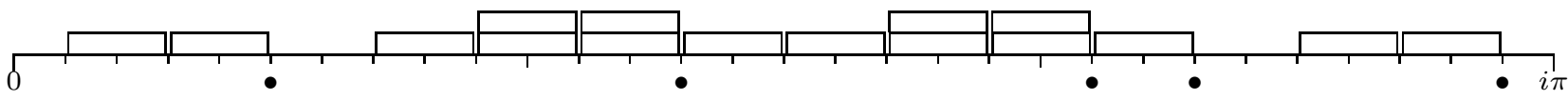

$S_{88}:$

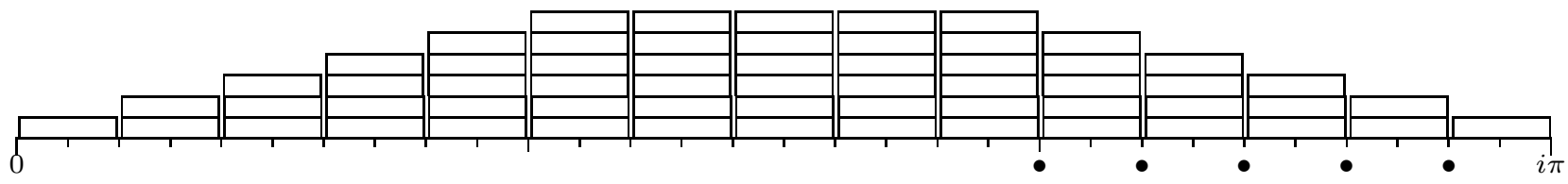

The rest of each picture, a stretch of wall running from (say) $i \pi$ to $2 i \pi$, can be obtained by reflecting the piece shown about the $\operatorname{line} \operatorname{Im}(\theta)=i \pi$, and then negating all the heights. Note that the heights are positive inside the physical strip, and negative outside - a general phenomenon that will be commented on at the end of this section. The positions of expected forward-channel poles (found from the three-point couplings and the masses) are shown by the symbols • below the axis - they occur precisely at the 'downhill' sections of wall (reading left to right). This apparent coincidence exemplifies a well-established relationship between the fusing structure and the nature of the pole residues, and will now be discussed.

The nature of the residue of any pole is easy to find from the corresponding picturel (the term residue being used somewhat loosely to mean the coefficient of the most singular

2 For the affine Toda S-matrices, this sort of block notation also allows the value of this residue, to leading order in $\beta$, to be identified; this was used in [5]. 
part). Blocks not contributing directly to a pole simply multiply its residue by a positive real number, and can be ignored (this is the reason for the minus sign in (2.12)). Furthermore, the contribution from two directly abutting blocks (one to the left and one to the right of the pole) is a positive multiple of $(+i) \cdot(-i)=1$, so these can also be ignored. Thus the nature of the residue is determined solely by the difference in the number of blocks immediately to the left and right of the pole, that is by the change in height of the wall of blocks at the position of the pole. The residues for the three simplest possibilities are

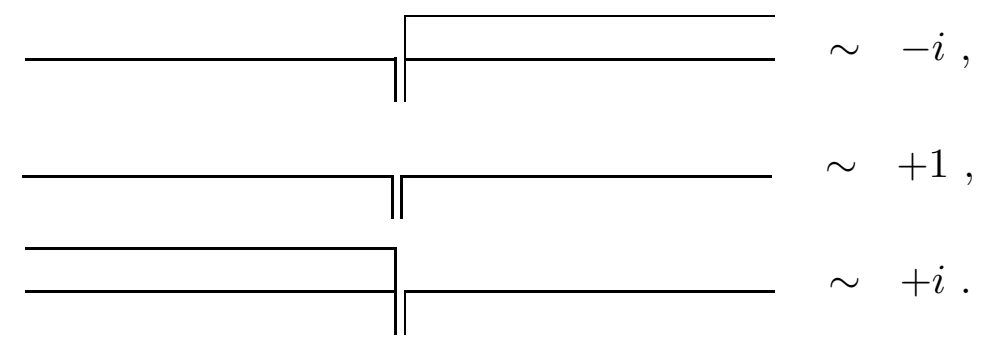

In cases $a$ and $c$, the total number of blocks to left and right of the pole is odd, and so the pole itself is of odd order. These odd order poles always have an interpretation in terms of the production of a bound state. Examination of the ADE scattering theories on a case-by-case basis has shown that case $c$, the downhill pole with a $+i$ residue, is always forward channel, while case $a$, uphill, is crossed channel. The pictures give a simple 'uphill/downhill' mnemonic by which to decide if an odd-order pole corresponds to a forward channel bound state, in agreement with what has already been observed in the two $E_{8}$ examples. (Note, though, that it remains unclear from the point of view of perturbation theory why the $+i$ residue should be forward channel for the higher odd-order poles. The mechanism by which this rule is reproduced, even for the third-order poles, is quite complicated [5].)

Another 'empirical' observation can be made: apart from the occasional exception at the very edge of the physical strip (for example, at 0 and $i \pi$ in $S_{88}$ above) the wall height never changes by more than \pm 1 . In the exceptional cases, the height change is always from -1 to 1 or back. Wall segments of negative height have zeroes instead of poles, so there is a cancellation and S-matrix is analytic at these points (and in fact is, by unitarity, forced to be equal to \pm 1 ). Hence (3.1) turns out to cover all possibilities for S-matrix poles. In particular, even-order poles always have positive real residues.

It might be expected that all the observations described above should have a universal explanation in the context of root systems. In fact, this can be achieved using only the most elementary properties of the simply-laced roots. 
Referring back to (2.19), consider the pole in $S_{i j}$ at relative rapidity $\frac{\pi i}{h} u\left(\phi_{i}, \alpha_{(j)}\right)$ (with $0<u\left(\phi_{i}, \alpha_{(j)}\right)<h$ for the physical strip). The two blocks contributing to this pole involve $\alpha_{(j)}$ and $w \alpha_{(j)}$, that involving $w \alpha_{(j)}$ being to the left (recall from (2.13) that $\left.u\left(\phi_{i}, w \alpha_{(j)}\right)=u\left(\phi_{i}, \alpha_{(j)}\right)-2\right)$. Thus the change $\delta h$ in wall height at this pole is given by

$$
\delta h=\left(\lambda_{i}, \alpha_{(j)}\right)-\left(\lambda_{i}, w \alpha_{(j)}\right) .
$$

Using (2.2) for the second equality, this simplifies:

$$
\delta h=\left(\left(1-w^{-1}\right) \lambda_{i}, \alpha_{(j)}\right)=\left(\phi_{i}, \alpha_{(j)}\right) .
$$

Being the inner product of two roots (of a simply-laced algebra), it is now clear that the change in wall height, if not zero, can only be \pm 1 or \pm 2 . These possibilities can be examined in turn.

A change of -1 should correspond to a bound state. But $\left(\phi_{i}, \alpha_{(j)}\right)=-1$ implies that $\phi_{i}+\alpha_{(j)}$ is a root, $-\alpha_{(k)}$ say, since it is just the Weyl reflection of $\phi_{i}$ with respect to $\alpha_{(j)}$. This gives a root triangle $\left\{\phi_{i}, \alpha_{(j)}, \alpha_{(k)}\right\}$ and from the fusing rule a non-zero threepoint coupling $C^{i j k}$. The fusing angle for the $\bar{k}$ bound state is, by (2.15), $\frac{\pi}{h} u\left(\phi_{i}, \alpha_{(j)}\right)-$ exactly that corresponding to the pole under discussion. Conversely if there $i s$ a threepoint coupling such that a bound state at the relevant fusing angle is a possibility, then $\delta h=-1$ is forced (since $\phi_{i}+\alpha_{(j)}=-\alpha_{(k)}$ is then a root, and so $\delta h=\left(\phi_{i}, \alpha_{(j)}\right)=$ $\left.\frac{1}{2}\left(\alpha_{(k)}^{2}-\phi_{i}^{2}-\alpha_{(j)}^{2}\right)=-1\right)$.

The story for $\delta h=1$ is similar, with the conclusion that $\delta h=1$ if and only if there is a bound state in the crossed channel.

These two cases have taken up both forward and crossed channels, so an even-order pole can never have an associated single-particle bound state. It only remains to remark that $\delta h= \pm 2$ implies $\phi_{i}= \pm \alpha_{(j)}$, and a relative rapidity for the putative pole of 0 or $i \pi$, the first to be found in $i i$ scattering, the second in $i \bar{\imath}$. As already mentioned, such 'poles' disappear by unitarity.

This then provides a universal explanation for the interplay between the bootstrap equations and the pole structure of the purely elastic S-matrices. In particular it elucidates the 'internal' consistency of these S-matrices, obeying as they do the very equations that they imply via their pole structure.

To close this section, a comment on a slightly simpler feature of (2.17) and (2.19), which also has an interpretation in terms of root systems. Over the full range from 0 to 
$2 \pi i$, the wall height can be both positive and negative, and indeed must be so to satisfy the unitarity constraint. But on physical grounds, the height had better not be negative for blocks in the physical strip: in such an eventuality, a perturbed conformal theory Smatrix would have zeroes in the physical strip, while the affine Toda theory would gain physical-strip poles with coupling-constant dependent positions. Now this height is given as the inner product of some root with a fundamental weight, and so is positive or negative according to whether the relevant root is positive or negative with respect to the given set of simple roots (to say this in another way, the wall height around $\frac{\pi i}{h}\left(u\left(\phi_{i}, \alpha_{(j)}\right)+1\right)$ is just one 'component' of the height of the root $\alpha_{(j)}$, namely that piece due to the simple root $\alpha_{i}$ ). Referring in particular back to (2.17), it is clear that the physical requirement reduces, traversing each orbit $\Gamma_{j}$ of the Coxeter element starting at the special root $\phi_{j}$, to a discussion of which roots are positive and which negative. Although the details will be omitted here, it is straightforward to use results (i), (ii) and (iii) from the beginning of section two, together with the implication from unitarity that approximately half 3 of the roots in a given orbit are positive, to see that the desired property of the wall heights does indeed follow from general theory.

\section{The signs of the three-point couplings}

In the perturbative treatment of the affine Toda theories, expansion of the potential to order $\beta$ results in a set of three-point couplings $C^{i j k}$ which, if non-vanishing, obey the 'area rule':

$$
C^{i j k}=\sigma^{i j k} \frac{4 \beta}{\sqrt{ } h} \Delta_{i j k}
$$

where $\Delta_{i j k}$ is the area of a triangle of sides $m_{i}, m_{j}$ and $m_{k}$ (the particle masses) and $\sigma^{i j k}$ is a phase of unit modulus, which given the hermiticity of the original Lagrangian can be taken to be plus or minus one. The vanishing/non-vanishing of the coupling is described by the rule (2.14). This, together with the normalisation of $C^{i j k}$, has now been derived in a general way [16]. However the signs $\sigma^{i j k}$ are a little more subtle. Clearly they can be changed around by negating some of the fields, but this does not mean that they can all be set to 1 . Indeed, cancellations necessary for perturbation theory to be compatible with integrability often depend crucially on the presence of relative phases between different

3 in fact exactly half, except for $A_{2 n}$ for which $h$ is odd and the situation is marginally more complicated. 
terms, which would not be present if all the $\sigma$ 's were equal to 1 (for some examples of this, see [5, 6]).

Despite the apparent arbitrariness involved, there is a special choice of normalisations which connects with the root system data already described. Recall that any three-point coupling $C^{i j k}$ results in a bound-state pole in $i j$ scattering of odd order $2 m+1$, and note that the field normalisations used in [4] can be altered so that in every case,

$$
\sigma^{i j k}=(-1)^{m}
$$

This is actually only a small increase in information over a formula given in [5], itself a consequence of a formula found by Braden and Sasaki [6]. Their result reads:

$$
\sigma^{i j k}=-\sigma^{i l \bar{m}} \sigma^{j m \bar{n}} \sigma^{k n \bar{l}}
$$

holding in this form whenever the triangle $\Delta_{i j k}$ is tiled internally by the three other mass/coupling triangles, $\Delta_{i l \bar{m}}, \Delta_{j m \bar{n}}$ and $\Delta_{k n \bar{l}}$. Note, (4.2) does not change with changes to the field normalisations. The consequence of this, remarked in [5], is that if $\Delta_{i j k}$ is tiled in a 'nested' fashion by $2 q+1$ other triangles $\left\{\Delta_{A}\right\}, A=1, \ldots 2 q+1$ (with phase factors $\left.\left\{\sigma^{A}\right\}\right)$, then

$$
\sigma^{i j k}=(-1)^{q} \prod_{A} \sigma^{A}
$$

(The tiling of a triangle is nested if it is tiled by three other triangles each of which is either untiled, or is itself tiled in a nested way. This allows (4.1) to be used inductively to derive (4.3).)

Now higher poles are also associated with tilings by mass triangles [5]. For an oddorder pole of order $2 m+1$, with $i j$ producing a bound state $\bar{k}$, the triangle $\Delta_{i j k}$ has a 'maximal' nested tiling (in fact, many such) by $2 m+1$ triangles, maximal in the sense that each constituent triangle cannot be further tiled. The number $2 m+1$ was called the depth of the coupling triangle $\Delta^{i j k}$ in [5]. Applying (4.3) then gives the phases for all coupling triangles as products of the phases for triangles of depth 1. It is then only necessary to check that all the unit depth phases can all be set to 1 to deduce (4.1) from (4.3).

Now (4.1), together with the discussion of the last section, can be used to give an expression for the signs in terms of the roots and weights. The physical strip pole for the $\bar{k}$ bound state in $i j$ scattering will be at $\frac{\pi i}{h} u\left(\phi_{i}, \alpha_{(j)}^{o}\right)$ for some $\alpha_{(j)}^{o} \in \Gamma_{j}(c f$ the discussion 
preceding (3.2)). Note, $\left\{\phi_{i}, \alpha_{(j)}^{o}, \alpha_{(k)}^{o}\right\}$, the corresponding root triangle for $C^{i j k}$, is oriented such that $0<u\left(\phi_{i}, \alpha_{(j)}^{o}\right)<h$. The order of the pole is

$$
2 m+1=\left(\lambda_{i}, \alpha_{(j)}^{o}\right)+\left(\lambda_{i}, w \alpha_{(j)}^{o}\right)
$$

Now the change $\delta h$ in the wall height at this point is -1 , as the pole is forward channel; so combining (4.4) with (3.2) gives

$$
m=\left(\lambda_{i}, \alpha_{(j)}^{o}\right)
$$

and $\sigma^{i j k}=(-1)^{\left(\lambda_{i}, \alpha_{(j)}^{o}\right)}$. This expression for $\sigma^{i j k}$ involves the choice of one particular root triangle, and furthermore its symmetry in $i, j$ and $k$ is not at all obvious. To remedy these defects, the idea of the orientation of a root triangle will have to be made a little more precise. Let $\left\{\alpha_{(i)}, \alpha_{(j)}, \alpha_{(k)}\right\}$ be any root triangle implying the nonvanishing of the coupling $C^{i j k}$; the orientation $\epsilon\left(\alpha_{(i)}, \alpha_{(j)}, \alpha_{(k)}\right)$ is then defined to be +1 if the projection into the $s=1$ subspace has a clockwise sense (going from $i$ to $j$ to $k$ ), and -1 if anticlockwise. Since $\epsilon\left(\alpha_{(i)}, \alpha_{(j)}, \alpha_{(k)}\right)=+1$ if and only if $0<u\left(\alpha_{(i)}, \alpha_{(j)}\right)<h$, the root triangle $\left\{\phi_{i}, \alpha_{(j)}^{o}, \alpha_{(k)}^{o}\right\}$ used above has orientation +1 . It will be helpful to define another quantity for (general) root triangles, namely

$$
f\left(\alpha_{(i)}, \alpha_{(j)}, \alpha_{(k)}\right)=\frac{1}{2}+\left(R \alpha_{(i)}, \alpha_{(j)}\right)
$$

where $R$, given by (2.3), has the important property that $R \phi_{i}=\lambda_{i}$. Finally, set

$$
m\left(\alpha_{(i)}, \alpha_{(j)}, \alpha_{(k)}\right)=\epsilon\left(\alpha_{(i)}, \alpha_{(j)}, \alpha_{(k)}\right) f\left(\alpha_{(i)}, \alpha_{(j)}, \alpha_{(k)}\right)-\frac{1}{2}
$$

For the original root triangle $\left\{\phi_{i}, \alpha_{(j)}^{o}, \alpha_{(k)}^{o}\right\}$, this coincides with (4.5) (this is the reason for the $\left.-\frac{1}{2}\right)$. But $m\left(\alpha_{(i)}, \alpha_{(j)}, \alpha_{(k)}\right)$ is the same for any triangle of roots for $C^{i j k}$, and furthermore is symmetrical in $i, j$ and $k$. To establish these properties, some more information on the set of root triangles is needed. There are in fact $2 h$ ordered triplets $\left\{\alpha_{(i)}, \alpha_{(j)}, \alpha_{(k)}\right\}$ for each non-zero three-point coupling (the ordering being needed to count correctly the cases when, say, $i=j$ ). Of these, $h$ can be found simply by acting 'diagonally' with powers of $w$ on an initial triangle. These all have the same orientation. To see that there are exactly $h$ more, consider the action of $\widetilde{w} \equiv-w_{\{\bullet\}}$ (one could equally well set $\widetilde{w}=w_{\{\circ\}}$; all the identities to be given below would be unchanged). As mentioned in [12], on the cosets $\widetilde{w}$ 
has the effect of two successive charge conjugations, that is no effect at all: $\widetilde{w} \Gamma_{i}=\Gamma_{i}$. But when acting on triangles, $\widetilde{w}$ reverses the orientation:

$$
\epsilon\left(\widetilde{w} \alpha_{(i)}, \widetilde{w} \alpha_{(j)}, \widetilde{w} \alpha_{(k)}\right)=-\epsilon\left(\alpha_{(i)}, \alpha_{(j)}, \alpha_{(k)}\right)
$$

Applying powers of $w$ to $\left\{\widetilde{w} \alpha_{(i)}, \widetilde{w} \alpha_{(j)}, \widetilde{w} \alpha_{(k)}\right\}$ then gives $h$ more possibilities, and the geometry of the projection into the $s=1$ subspace shows that there can be no more. (To be more precise, since $U_{i j}^{k}$ is fixed, (2.15) shows that once the root $\alpha_{(i)}$ has been chosen there are only two possible directions for the $s=1$ projection of $\alpha_{(j)}$. But the roots in a single orbit all project to different directions for $s=1$, so there are at most two roots in $\Gamma_{j}$ which can form a triangle involving $\alpha_{(i)}$ and a root from $\Gamma_{k}$. Letting $\alpha_{(i)}$ run through its orbit then gives a maximum of $2 h$ triangles.)

An important consequence of the above is that all triangles for a given coupling can be obtained from an initial one by acting with $w$ and $\widetilde{w}$. Now the effect of these two operations on the orientation has already been given: $w$ leaves $\epsilon$ invariant, while $\widetilde{w}$ negates it. It is also clear that $w$ leaves $f$ unchanged, $w$ being an orthogonal transformation which commutes with $R$. The action of $\widetilde{w}$ is a little harder to see, but the identity $\widetilde{w} R \widetilde{w}=1-R$ together with the fact that the inner product of $\alpha_{(i)}$ and $\alpha_{(j)}$ must be -1 (their sum being another root) implies

$$
f\left(\widetilde{w} \alpha_{(i)}, \widetilde{w} \alpha_{(j)}, \widetilde{w} \alpha_{(k)}\right)=-f\left(\alpha_{(i)}, \alpha_{(j)}, \alpha_{(k)}\right)
$$

Comparing with (4.8) establishes the invariance of $m\left(\alpha_{(i)}, \alpha_{(j)}, \alpha_{(k)}\right)$ under the diagonal action of both $w$ and $\widetilde{w}$, and hence its insensitivity to the choice of root triangle.

There remains the symmetry of (4.7) between $i, j$ and $k$. The orientation is completely antisymmetric in its arguments, so it will be enough to demonstrate that the same is true of $f$. First consider swapping $\alpha_{(i)}$ and $\alpha_{(j)}$ in (4.6). From (2.5), and using again that the inner product of $\alpha_{(i)}$ and $\alpha_{(j)}$ must be -1 , this sends $f$ to $-f$. Now consider a cyclic permutation of $\alpha_{(i)}, \alpha_{(j)}$ and $\alpha_{(k)}$. The three roots sum to zero, so $\left(R \alpha_{(j)}, \alpha_{(k)}\right)=$ $\left(R \alpha_{(j)},-\alpha_{(i)}-\alpha_{(j)}\right)=\left(R \alpha_{(i)}, \alpha_{(j)}\right)$, using (2.5) and also the fact that $\left(R \alpha_{(j)}, \alpha_{(j)}\right)=1$. Thus $f$ is unchanged by a cyclic permutation of its arguments, and this is enough to establish antisymmetry.

This completes the proof of the claims following equation (4.7). The expression can now be used in (4.1), it already having been mentioned that for one particular triangle (and hence for all) (4.7) coincides with the previously-derived (4.5). This can be used 
in a complete specification of the three-point couplings for the simply-laced affine Toda theories. With a simple rewriting of the resulting expression for $(-1)^{m}$, the three-point coupling data can be summarised as follows:

- $C^{i j k} \neq 0$ iff $\exists \alpha_{(i)}+\alpha_{(j)}+\alpha_{(k)}=0$ (where $\alpha_{(i)} \in \Gamma_{i}, \alpha_{(j)} \in \Gamma_{j}, \alpha_{(k)} \in \Gamma_{k}$ ).

- There is a normalisation of the fields such that in these cases

$$
C^{i j k}=\epsilon\left(\alpha_{(i)}, \alpha_{(j)}, \alpha_{(k)}\right)(-1)^{\left(R \alpha_{(i)}, \alpha_{(j)}\right)} \frac{4 \beta}{\sqrt{ } h} \Delta_{i j k}
$$

\section{A formula due to Klassen and Melzer}

In their investigations of the Thermodynamic Bethe Ansatz, Klassen and Melzer [9] observed an interesting universal feature of the minimal purely elastic S-matrices. If the matrix $N_{i j}$ is defined by

$$
N_{i j}=-\frac{1}{2 \pi i}\left[\ln S_{i j}(\theta)\right]_{\theta=-\infty}^{\theta=\infty}
$$

then for the minimal (perturbed conformal field theory) S-matrices,

$$
N=2 C^{-1}-I
$$

where $C$ is the relevant (non-affine) Cartan matrix, and $I$ the unit matrix. Using this result, Klassen and Melzer were able to calculate the central charges of the ultra-violet limits of these theories, finding agreement with the idea that these S-matrices do indeed describe perturbations of certain conformal field theories. Such issues will not be the concern here; the purpose of this section is merely to give a simple and universal proof of (5.2) starting from the general S-matrix expression of [12].

Klassen and Melzer used unitary blocks $f_{\alpha}(\theta) \equiv(h \alpha)(\theta)$, in terms of which the Smatrix element $S_{i j}$ was written

$$
S_{i j}=\prod_{\alpha \in A_{i j}} f_{\alpha}(\theta)
$$

The parameter $\alpha$ was taken to satisfy $-1<\alpha \leq 1$, and (5.1) reduced to

$$
N_{i j}=\sum_{\alpha \in A_{i j}}(1-|\alpha|) \operatorname{sgn}(\alpha)
$$

with the convention that $\operatorname{sgn}(0)=0$ ( since $\left.f_{0} \equiv 1\right)$. It will be more convenient below to choose a different range for $\alpha$, namely $0 \leq \alpha<2$. Using $f_{\alpha}=f_{\alpha+2}$ to relabel the 
negatively-indexed blocks, together with the fact that $(1-|\alpha|) \operatorname{sgn}(\alpha)=1-(\alpha+2)$ for $-1<\alpha<0$, equation (5.3) becomes

$$
N_{i j}=\sum_{\alpha \in A_{i j}}(1-\alpha)-\left|A_{i j} \cap\{0\}\right|,
$$

where now $0 \leq \alpha<2$, and the second term undoes the overcounting in the first whenever $\alpha=0$.

To evaluate (5.4), it is simplest to use the first expression of (2.27). Each unitary block $\left(2 p+u_{i j}\right)$ corresponds to an $\alpha \in A_{i j}$ equal to $\left(2 p+u_{i j}\right) / h$, while $\left|A_{i j} \cap\{0\}\right|$ is simply the number of blocks (0). Such blocks will only be found if $u_{i j}=0$, and so $\left|A_{i j} \cap\{0\}\right|=0$ if $i$ and $j$ have different colours. Otherwise, $(0)$ is raised to the power $\left(\lambda_{i}, \phi_{j}\right)$. For the the ordering $(2.7)$ of the simple roots, the full set of these inner products is

$$
\begin{array}{ll}
\left(\lambda_{\bullet}, \phi_{\bullet^{\prime}}\right)=\delta_{\bullet \bullet '} & \left(\lambda_{\circ}, \phi_{\bullet \prime}\right)=-C_{\circ \bullet} \\
\left(\lambda_{\bullet}, \phi_{\circ^{\prime}}\right)=0 & \left(\lambda_{\circ}, \phi_{\circ^{\prime}}\right)=\delta_{\circ \circ^{\prime}}
\end{array}
$$

Hence the general result is $\left|A_{i j} \cap\{0\}\right|=\delta_{i j}$, and $N_{i j}$ is given by

$$
N_{i j}=\sum_{p=0}^{h-1}\left(1-\left(2 p+u_{i j}\right) / h\right)\left(\lambda_{i}, w^{-p} \phi_{j}\right)-\delta_{i j}
$$

Strictly speaking, the fact that $u_{\bullet}=-1$ means that if $i$ is of type $\bullet$ and $j$ of type $\circ$, the first block counted by (5.6) corresponds to $\alpha=-1 / h$, outside the desired range. This can be ignored as the power to which this block is raised, $\left(\lambda_{i}, \phi_{j}\right)$, is zero here by (5.5).

Making use of equations (2.4) and (2.6),

$$
N_{i j}=\left(\lambda_{i},\left(\left(h-u_{i j}\right) P_{0}+2 R\right) \phi_{j}\right)-\delta_{i j}=2\left(\lambda_{i}, \lambda_{j}\right)-\delta_{i j}
$$

the results $P_{0}=0$ ( 0 is never an exponent) and $R \phi_{j}=\lambda_{j}$ giving the second equality. Since $\left(\lambda_{i}, \lambda_{j}\right)$ is exactly the inverse of the Cartan matrix, equation (5.2) now follows immediately.

\section{Conclusions}

Various previously-observed features of the ADE purely elastic scattering theories are now known to follow from general principles, especially given recent work on affine Toda perturbation theory [16] and the Clebsch-Gordan rule for fusings [17. With regards to the S-matrices, a notable feature of the treatment given above, as compared to that in [12], is 
that the splitting of the roots according to type (colour) has become much less important. Essentially, once the notations (2.13) have been set up, this distinction can be forgotten. All that is needed is equation (2.15), relating the quantities $u(\alpha, \beta)$ to the fusing angles. However, it should not be forgotten that the splitting of the particles into two sets does express a geometrical property of the projections of their orbits, reflected in the fact [12] that two particles of the same type always fuse at an even multiple of $\pi / h$, two particles of opposite type at an odd multiple of $\pi / h$. Hence the split still has physical implications, even if these are best left hidden for most calculations.

As regards future work, there are two obvious questions to ask. Within the context of theories with diagonal S-matrices, are there any other physically reasonable possibilities beyond those associated with the ADE series? To formulate this question properly, it must be decided exactly what is meant by 'physically reasonable'. For example, whenever a subalgebra of the fusing algebra can be formed (such as emerges when a twisted folding is considered [4] [ []), a self-consistent set of S-matrix elements, obeying the bootstrap equations that they imply via their odd-order poles, can be obtained simply by taking the corresponding submatrix of the 'parent' S-matrix. However, in such cases there are always higher-order physical poles which are inexplicable without the full set of particles of the parent theory, forcing their re-inclusion and returning the theory to the ADE set. (A similar phenomenon allows the solitons of the sine-Gordon theory to be inferred from the S-matrix elements of the breathers alone 18].) The only other purely elastic S-matrices that seem to have been discussed so far [19,20] all obey rather different self-consistency requirements, in that the prescription for assigning forward and crossed channels to the odd-order poles is changed. Such conditions appear to be appropriate for theories with non-hermitian lagrangians [19], and so these S-matrices should in any case fall outside an initial (perhaps ADE) classification of unitary purely elastic scattering theories. Nevertheless, it would be interesting if they could also be given an interpretation in terms of root systems.

The second question concerns the relevance of any of the above to theories with multiplets and non-diagonal S-matrices. This would require some similarity in structure between the bootstraps for the purely elastic scattering theories and those for at least a subset of the more complicated models. At least at the level of the fusings, the same structure has emerged in the study of perturbations of $N=2$ supersymmetric conformal theories [15] (although in fact not for all the ADE series). Other possible candidates for inclusion in the subset include the principal chiral models [21]. S-matrices with the 
same scalar (CDD) part have been proposed for perturbations of certain conformal field theories [22]. There are many coincidences (for example, of mass spectra) and even some explicit calculations [23] suggesting that a connection may indeed be found, but the greatlyincreased complexity of the bootstrap equations makes a complete analysis difficult.

\section{Acknowledgements}

I would like to thank Ed Corrigan for interesting discussions and comments on the manuscript, and the Research Institute for Mathematical Sciences, Kyoto University for their kind hospitality while this work was being completed. The work was supported in part by a grant of the British Council. I am grateful to the Royal Society for a Fellowship under the European Science Exchange Programme. 


\section{References}

[1] A. B. Zamolodchikov, "Integrable Field Theory from Conformal Field Theory", Proceedings of the Taniguchi Symposium, Kyoto (1988);

A. B. Zamolodchikov, Int. J. Mod. Phys. A4 (1989) 4235.

[2] V. A. Fateev and A. B. Zamolodchikov, Int. J. Mod. Phys. A5 (1990) 1025.

[3] A. E. Arinshtein, V. A. Fateev and A. B. Zamolodchikov, Phys. Lett. B87 (1979) 389.

[4] H. W. Braden, E. Corrigan, P. E. Dorey and R. Sasaki, Nucl. Phys. B338 (1990) 689.

[5] H. W. Braden, E. Corrigan, P. E. Dorey and R. Sasaki, Nucl. Phys. B356 (1991) 469.

[6] H. W. Braden and R. Sasaki, Phys. Lett. B255 (1991) 343.

[7] P. Christe and G. Mussardo, Nucl. Phys. B330 (1990) 465;

P. Christe and G. Mussardo, Int. J. Mod. Phys. A5 (1990) 4581.

[8] C. Destri and H. J. de Vega, Phys. Lett. B233 (1989) 336.

[9] T. R. Klassen and E. Melzer, Nucl. Phys. B338 (1990) 485.

[10] See for example A.B.Zamolodchikov and Al.B.Zamolodchikov, Ann. Phys. 120 (1979) 253.

[11] M. Karowski, Nucl. Phys. B153 (1979) 244.

[12] P. E. Dorey, Nucl. Phys. B358 (1991) 654.

[13] B. Kostant, Am. J. Math. 81 (1959) 973.

[14] N. Bourbaki, Groupes et algèbres de Lie IV, V, VI, (Hermann, Paris 1968): VI, 1, exerc. 22 .

[15] W. Lerche and N.P. Warner, Nucl. Phys. B358 (1991) 571.

[16] M. D. Freeman, Phys. Lett. B261 (1991) 57;

A. Fring, H. C. Liao and D. Olive, 'The mass spectrum and coupling in affine Toda theories' preprint IC/TP-90-91/25.

[17] H. W. Braden, 'A note on affine Toda couplings', Edinburgh preprint 91-01.

[18] C. J. Goebel, Prog. Theor. Phys. Suppl. 86 (1986) 261.

[19] J. Cardy and G. Mussardo, Phys. Lett. B225 (1989) 275.

[20] P. G. O. Freund, T. Klassen and E. Melzer, Phys. Lett. B229 (1989) 243.

[21] E. Ogievetsky and P. Wiegmann, Phys. Lett. B168 (1986) 360.

[22] D. Bernard and A. LeClair, "Quantum group symmetries and non-local currents in 2D QFT", preprint CLNS-90/1027, SPhT-90/144, Comm. Math. Phys. to appear.

[23] N. J. MacKay, Nucl. Phys. B356 (1991) 729. 